\title{
Picosecond Ultrasonic Microscopy of Semiconductor Nanostructures
}

\author{
Thomas J GRIMSLEY ${ }^{1}$, Cuong H DANG ${ }^{1}$, Qiang ZHANG ${ }^{2}$, Shan CHE ${ }^{1}$, \\ Fan YANG ${ }^{1}$, G Andrew ANTONELLI ${ }^{1}$, Arto V NURMIKKO ${ }^{1,2}$, Humphrey J \\ MARIS $^{1}$ \\ ${ }^{1}$ Department of Physics, Brown University \\ Providence, RI, U.S.A.; Phone: 1401863 2642, Fax 1401 2024; thomas_grimsley@brown.edu: \\ cuong_dang@brown.edu, shan_che@brown.edu, fan_yang@brown.edu, george_antonelli@brown.edu, \\ humphrey_maris@brown.edu \\ ${ }^{2}$ Division of Engineering, Brown University \\ Providence, RI, U.S.A.; qiang_zhang@brown.edu, arto_nurmikko@brown.edu
}

\begin{abstract}
We report on a picosecond ultrasonics study of nanostructures by high frequency ultrasound. A sound pulse is generated when an ultra short laser pulse is absorbed in a metal transducer film. The sound propagates across a thin layer (0.5-2 microns) of water and is then reflected from the surface of the sample being examined. The efficiency of optoacoustic detection of the reflected sound is enhanced through the use of a resonant optical cavity. We report on experiments in which sound is reflected from patterned nanostructures. In these experiments we are able to study the propagation of sound down narrow sub-100 nm channels.
\end{abstract}

Keywords: Picosecond Ultrasonics, Optical Cavity, Sound Propagation, Nanostructures

\section{Introduction}

The technique of picosecond ultrasonics [1] provides a way to generate short ( 1 ps) acoustic pulses for fundamental studies and metrology of small structures. An ultrashort pump light pulse is absorbed at the surface of a material, and the optical reflectivity of the sample is then measured with a probe pulse whose arrival is delayed with respect to the pump pulse. The pump pulse excites the sample and generates an elastic stress; as this stress relaxes it launches an acoustic pulse into the sample. The technique of picosecond ultrasonics has been used to study the vibrational modes of nanostructures directly [2]. Picosecond ultrasonics has also been used to generate sound pulses and scatter them off of a semiconductor nanostructure [3]. In this paper we demonstrate an acoustic microscope in which sound waves are generated optically, transmitted through a coupling fluid (water), and detected after reflection from siliconbased test samples which feature periodic nanoscale deep trenches.

\section{Pulsed Acoustic Microscopy and Picosecond Ultrasonics}

In a conventional acoustic microscope sound waves are generated by an electromechanical transducer, and brought to a focus at the surface of the sample which is immersed in a fluid coupling medium. Since the demonstration of a scanning acoustic microscope by Lemons and Quate [4], many researchers have explored the possibilities of acoustic microscopy. Using water as the coupling medium between the transducer and the sample, a resolution on the order of optical wavelengths has been demonstrated [5]. One of the appeals of using sound to image materials is the possibility of detecting subsurface defects in materials that are opaque optically, but relatively transparent (i.e., non-attenuating) acoustically. In addition, an acoustic microscope can image systems 
where the optical contrast is small but the acoustic contrast, or the difference in mechanical properties, is high [6].

In pulsed acoustic microscopy, the spatial resolution is fundamentally limited by the attenuation of the high frequency components of the sound pulse [6]. In a linear viscoelastic fluid, the attenuation $\alpha$ of sound is proportional to the square of the frequency $f$ [7], i.e., $\alpha=A f^{2}$. As a result, after a sound pulse that is initially very narrow in space propagates a distance $x$, it will take on a Gaussian shape

$$
\eta(x)=A_{0} \exp \left(-X^{2} / \sigma^{2}\right),
$$

where $X$ is the spatial distance from the centre of the pulse,

$$
\sigma=\frac{v}{\pi} \sqrt{A x}
$$

and $v$ is the sound velocity. For a pulse that has travelled through 1 micron of water at room temperature, a pulse of negligible initial width will broaden to a full width at half maximum of about $117 \mathrm{~nm}$. Hence, to reduce this broadening (and improve microscope resolution) due to attenuation it is important to use a lens with a small radius of curvature so as to shorten the sound path. As the radius of curvature of the lens decreases, shorter acoustic pulses are required since the spatial length of the sound must be less than the round trip path from the lens to the sample. Such short pulses can readily be generated and detected using picosecond ultrasonic techniques.

\section{Planar Opto-Acoustic Microscopy}

In this work, we introduce new methods for improving acoustic microscopy. We report here on proof-of-principle experiments where, instead of using focused ultrasound, we send a planar sound pulse at the sample. A large area detector is used to sense the returning sound acousto-optically. Information about the geometry of the sample is obtained from the time-dependence of the detected signal. The experiment is shown in simplest form in Fig. 1. A picosecond light pulse is absorbed at the surface of the transducer and a planar sound pulse is generated. This pulse propagates to the sample through the water coupling fluid. Part of the pulse is reflected at the top of the sample and part travels to the bottom of the trenches and is reflected there. From the difference between the arrival times of the echoes coming from the top of the sample and from the bottom of the trenches the depth of the trenches can be determined.

For any acoustic microscope to be effective, it is important that the measurement time be made as short as possible, i.e., there must be a good signal to noise ratio. This is difficult to achieve because of the large impedance mismatch between the solid material in which the sound is generated and detected and the coupling fluid. For microscopes in which the acoustic pulse has Fourier components lying in a narrow range around a carrier frequency, an antireflection coating can be applied to enhance the sound transmission. However, the temporal pulse shape generated in picosecond ultrasonics has a broad Fourier spectrum and so antireflection coatings are not effective. As a result it is necessary to find other means to enhance the detection sensitivity to compensate for the loss of amplitude when the sound enters and leaves the coupling fluid.

In the work reported here, we have used a standard pump and probe optical system but have added an optical cavity (resonant at probe laser wavelength) to increase the 


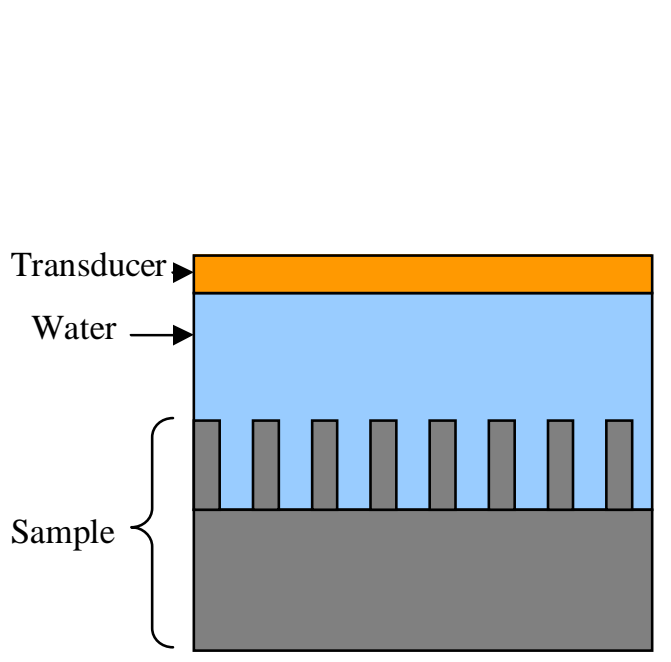

Figure 1. Schematic of experimental geometry with a nanostructure target sample

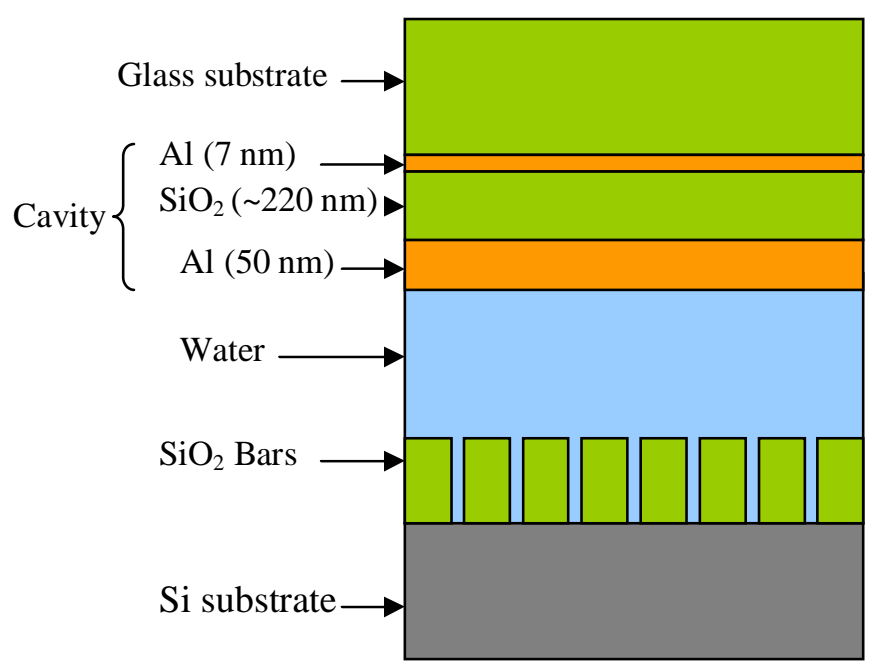

Figure 2. Addition of an optical cavity for enhancing optoacoustic sensitivity. The thickness of the water film is $0.5-3.0$ microns

acousto-optic detection sensitivity (Fig. 2). The cavity acts as a planar $\lambda / 2$ Fabry-Perot resonator, and the $\mathrm{SiO}_{2}$ film thickness is chosen such that the cavity has a mode near the $800 \mathrm{~nm}$ wavelength of our sub picosecond pulse laser source. When the returning sound pulse enters the structure and changes the thickness of the $\mathrm{SiO}_{2}$ layer, the resonance of the cavity is shifted spectrally resulting in a large change in the optical reflectivity. Note that the cavity also enhances the initial generation of sound since near resonance the absorption of light in the cavity is larger than the absorption that takes place at the surface of a single metal film. Both aluminium films are acoustic sources.

One disadvantage of the cavity technique is that there are certain frequencies of sound that generate a very small change in reflectivity. The first frequency where the cavity has a minimum sensitivity is when the wavelength of the sound is equal to the thickness of the cavity layer. When this happens, the integral of the strain in the cavity vanishes. For a cavity thickness of $220 \mathrm{~nm}$ and a sound velocity of $6 \mathrm{~nm} \mathrm{ps}^{-1}$, the first frequency is about $30 \mathrm{GHz}$. In practice, this does not result in a significant problem because the returning sound pulse has a frequency spectrum that does not extend up to this frequency.

\section{Results}

We examined several silicon-based semiconductor structures with the optical-cavity equipped picosecond ultrasonic setup. The first sample was an unpatterned silicon wafer whose surface roughness was measured to be $\sim 0.3 \mathrm{~nm}$. The change in reflectivity $\Delta R$ of the laser probe pulses as a function of the pump-probe time delay is shown in Fig. 3 for a series of water film thicknesses. The initial fast oscillations at earlier times are a consequence of optical scattering off a sound wave travelling through the glass substrate (Brillouin oscillations), while the slowly varying background is due to a combination of effects, such as the cooling of various films of the cavity. The water film thickness can be calculated using the arrival times of the pulses and the sound velocity in water $(1.5$ $\mathrm{nm} \mathrm{ps}^{-1}$ ). (These types of measurements can be used to measure the frequency dependence of ultrasound attenuation in the $\sim 10 \mathrm{GHz}$ range; to be reported elsewhere). 


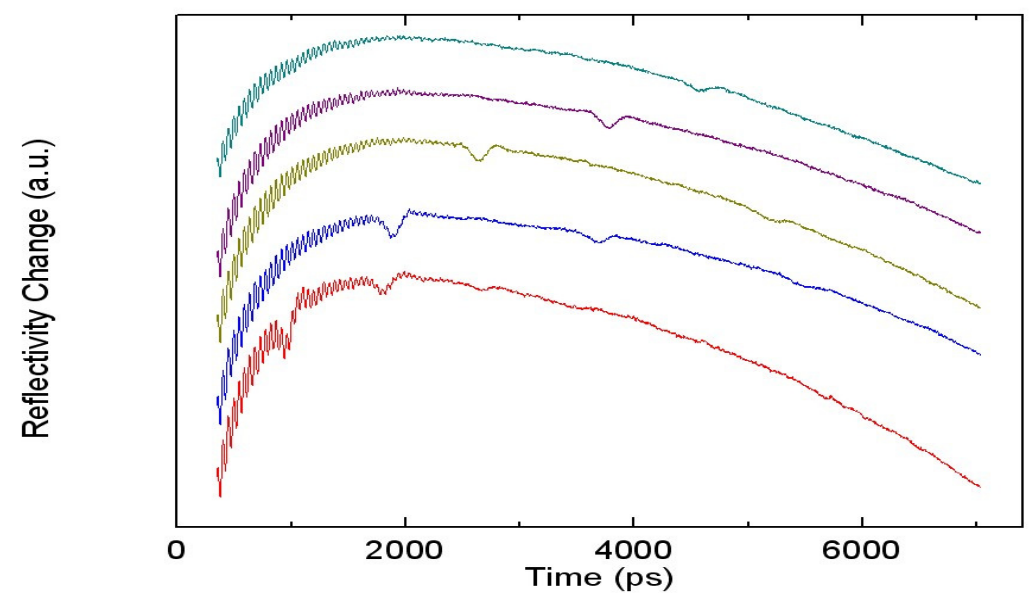

Figure 3. Change in reflectivity as a function of the time delay of the probe beam for an unpatterned silicon sample. The water film thicknesses, from top to bottom, are $3.35,2.76,1.93,1.35$, and 0.64 microns

A series of nanostructured microelectronics test samples that had high precision lithographically-defined silicon dioxide lines deposited on a silicon substrate were investigated. Top down and side view SEM images of a section of sample 1 are shown in Fig. 4. The dark lines are the trenches between the lines. The repeat spacing is about $250 \mathrm{~nm}$, and so for an optical pump spot 10 microns in diameter multiple trenches are examined at once. The depth of the trenches is $400 \mathrm{~nm}$. The change in optical reflectivity as a function of probe delay time is shown in Fig. 5. The arrival time of the first echo, labelled (a) in Fig. 5, we identify as the sound bouncing off the tops of the silicon dioxide lines and corresponds to a water film thickness of 1.30 microns. The echo labelled (b) corresponds to the sound that has travelled down the trench and reflected off the bottom. The difference between the arrival times of (a) and (b) corresponds to the round trip time through the trench. Using the sound velocity, we find that the trench depth is $410 \mathrm{~nm}$ with an uncertainty of $10 \mathrm{~nm}$. The difference between the arrival times of (a) and (c) corresponds to a thickness of 1.7 microns of water, which suggests that the dip at (c) is caused by sound that has reflected off the top once and the bottom once. The difference between the arrival times of (c) and (d) corresponds to the round trip time through $\sim 410 \mathrm{~nm}$ of water, which suggests that dip (d) is due to sound that has reflected off of the bottom twice.

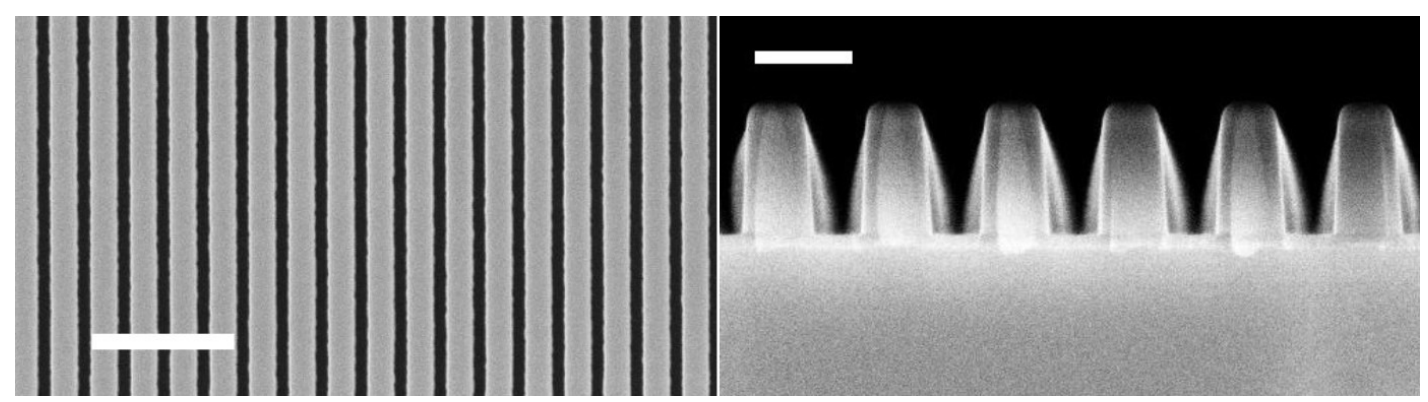

Figure 4. Left: Top view SEM image of silicon dioxide lines on a silicon substrate. The scale bar is 1 micron. Right: Side view SEM image of the sample cleaved along a plane perpendicular to the lines. Scale bar is $300 \mathrm{~nm}$ long and the trench depth is about $400 \mathrm{~nm}$.

The trenches in sample 1 had a depth-to-width aspect ratio of $\sim 5$. In Fig. 6 we show an SEM image and data taken on trenches with an aspect ratio of 7.5 . Again, the dip in 


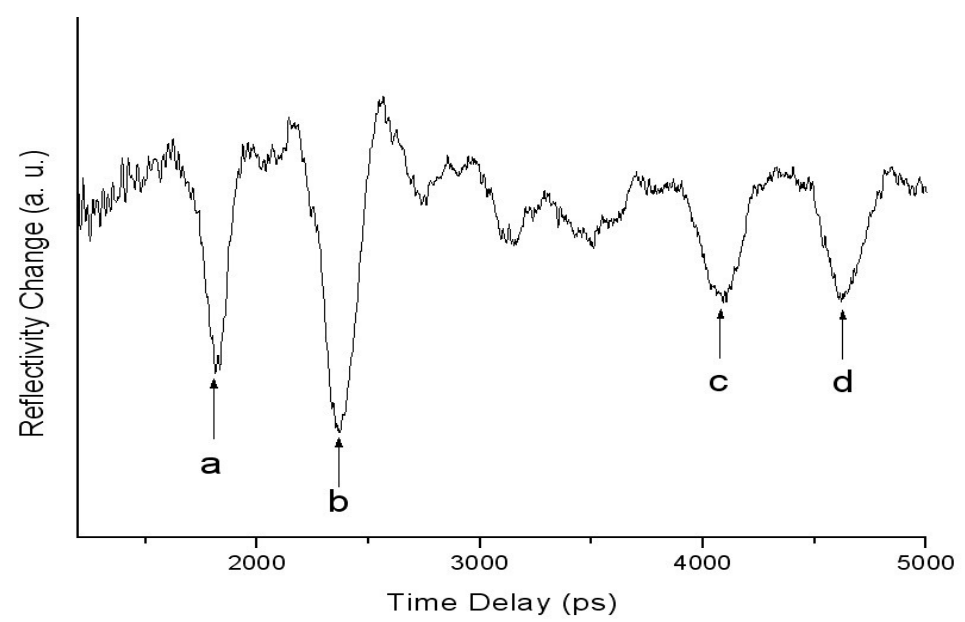

Figure 5. Change in optical reflectivity as a function of probe pulse time delay for sample 1. See text for description of echoes a-d.

the reflectivity labelled (a) is the first echo from the tops of the lines, while (b) is from the bottom of the trenches. The difference in the arrival times corresponds to the round trip time through $430 \mathrm{~nm}$ of water, with an uncertainty of $20 \mathrm{~nm}$. Surprisingly, while about $80 \%$ of the total area of sample 2 is covered by the silicon dioxide mesas, the magnitude of the echo from the bottom of the trench is nevertheless comparable to that from the top of the trenches. This suggests that the acoustic pulse is being scattered from the nanoscale details of the sample profile in a complex manner, and that the technique may offer further opportunities in high precision metrology of such structures beyond any conventional technique such as optical microscopy, for example.

From the SEM images in Fig. 4, the area presented to the incoming acoustic pulses is $65 \%$ mesas, and $35 \%$ trenches. However, from Fig. 5 it is clear that echo (b) is larger than echo (a) indicating that the sound has been "funnelled" down into the trenches. Also, in between the set of echoes (a)\&(b) and (c)\&(d) is some additional temporal structure, again suggesting that the trenches are scattering the sound in ways that cannot be accounted for by a simple ray model. Finally, the width of the acoustic pulse when it reaches the sample is on the order of the pitch of the sample, so that added contributions by complicated scattering of ultrasound can be expected.
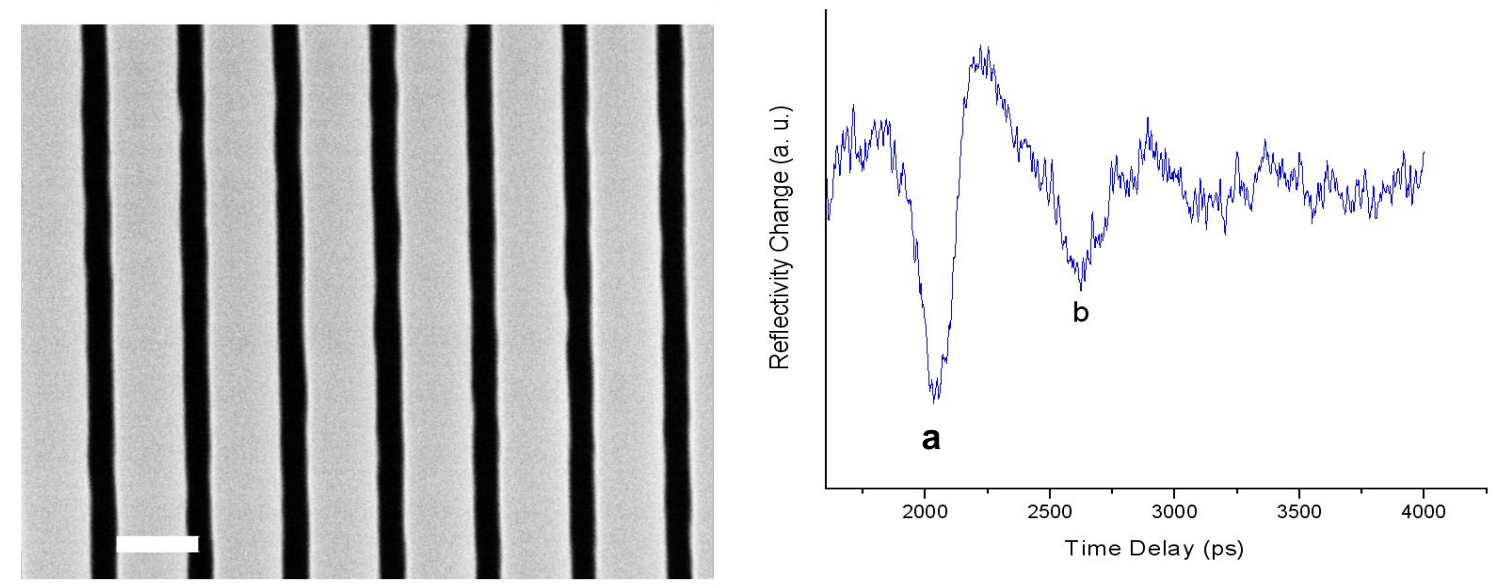

Figure 6. Left: Top down SEM image of sample 2. The scale bar is $200 \mathrm{~nm}$. The pitch is $\sim 250 \mathrm{~nm}$ and the trench width $\sim 55 \mathrm{~nm}$. Right: Change in optical reflectivity as a function of probe pulse time delay for sample 2. See text for description of (a) and (b). 

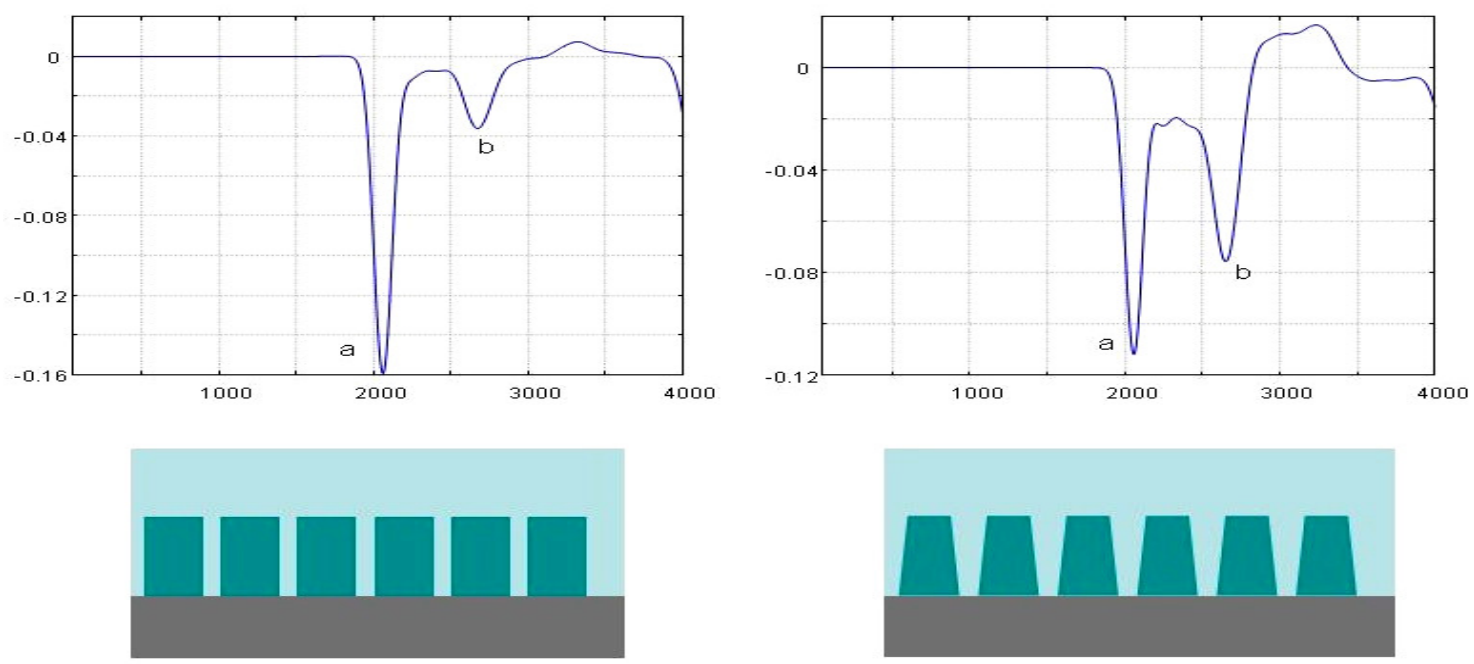

Figure 7. Simulated signal at transducer faces for sample 2. Left: Trenches assumed to be rectangular. Right: Trenches wider at the top. The simulation shows the pressure on the transducer face as a function of time (ps).

In order to explore the connection between sample profile and the observed echo pattern we have used a finite-difference method to simulate the signal. In Fig. 7 the results of simulations for two different profiles of the trenches are presented. We see that a slight change in the surface profile results in a large change in the relative height of the echo from the top of the sample and the echo from the bottom of the trench. (Such differences are important elements of quality control in the first stages of fabrication of state-of-the-art microelectronic circuits). This indicates that the technique of planar optoacoustic microscopy is able to measure profile characteristics of nanostructures as well as trench depth. We are currently exploring these opportunities in more detail to develop techniques to relate features in the echo pattern to details of sub-100 nm nanostructures. In addition, we are developing a focusing scanning version of this picosecond optoacoustic measurement system.

\section{References}

1. C. Thomsen, H. T. Grahn, H. J. Maris and J. Tauc, 'Surface Generation and Detection of Phonons by Picosecond Light Pulses', Phys. Rev. B, 34, pp 4129-38, 1986.

2. G.A. Antonelli, S.G. Malhotra, J.E.M. Harper, and H. J. Maris, 'Picosecond Ultrasonics Study of the Vibrational Modes of a Nanostructure', J. Appl. Phys., 91 (5), pp 3261-7, 2002.

3. B. C. Daly et al, 'Imaging Nanostructures with Coherent Phonon Pulses', Appl. Phys. Lett., 84 (25), pp 5180-5182, 2004.

4. R. A. Lemons and C. F. Quate, 'Acoustic Microscope - a Scanning Version', Appl. Phys. Lett., 24 (4), pp 163-5, 1974.

5. V Jipson and C. F. Quate, 'Acoustic Microscope at Optical Wavelengths', Appl. Phys. Lett., 32 (12), pp 789-91, 1978.

6. A. Briggs, 'Acoustic Microscopy - a Summary', Rep. Prog. Phys., 55, pp 851909, 1992.

7. A. B. Bhatia, Ultrasonic Absorption, Oxford University Press, Oxford, 1967. 